\title{
The Role of AI in Battling Against Covid-19 Crisis in India
}

\author{
Shakira Fathima $\mathrm{H}^{\mathrm{a}, 1}$ and Dilshad Begum $\mathrm{M}^{\mathrm{a}}$ \\ ${ }^{a}$ Assistant Professor, Department of Computer Science, JBAS College for Women, \\ Chennai, India
}

\begin{abstract}
The impact of Covid-19 outbreak has become a matter of grave concern in India. The scarcity of resources to endure the epidemic outbreak combined with the fear of overburdened health care systems had forced many Indian states to go for a partial or complete lockdown. Stimulated by the need for employing Artificial Intelligence (AI) in battling Covid-19 crisis, this paper highlights the importance of $\mathrm{AI}$ in responding to the Covid-19 outbreak and curtail the severity of the disease. Artificial Intelligence (AI) aim is to open the door for human in the prediction of the novel Corona Virus disease. Researchers are in process of using AI in finding new drugs and medicines for Covid cure and focusing on detection and analysis of infectious patients, through AI based Machine learning (ML) Algorithms and Expert Systems. Owing to huge number of cases, Covid-19 remains a global health problem, Al could take individual conditions into account, produce suitable decisions and promise to make great strides in managing Corona Virus. We begin with an outline of AI, identify the various applications aimed at fighting the disease, then highlight the challenges and issues associated with the cutting edge solutions finally arriving on the recommendations for the communication to effectively control the Covid-19 crisis.
\end{abstract}

Keywords. Artificial Intelligence, Covid-19, Detection and Analysis, Machine learning, Expert Systems.

\section{Introduction}

Artificial intelligence (AI) and Expert Systems that mimic human beings, has not only peeked in business and society but has also given a deep footprint in the field of healthcare. These technologies have proven to give an upper mark of support to transform many aspects of patient care, as well as administrative processes within healthcare organization [1]. AI is never tired, it does not get frustrated even during the pressurized situations, which are some of the most critical benefits while working with a problem of such complexity. There are already a number of research studies in process which suggest that AI can perform better than humans at key healthcare tasks such as disease diagnosis, appropriate medicinal support etc. AI has also tagged an impression in battling against the recent global crisis of Covid-19 [2,3]. In this baffling battle, AI is playing a vital role in fighting against the disease in various ways like facial recognition cameras to track the

\footnotetext{
${ }^{1}$ Shakira Fathima H, Department of Computer Science, JBAS College for Women, Chennai, India.

E-mail: shakirafathima.h@jbascollege.edu.in.
} 
infected patients with travel history, robots to deliver food and medicines, drones to disinfect public places, to patrol and broadcast audio messages to public encouraging them to stay at home etc. Many researches using AI are also in progress to find new drugs and medicines for the Covid cure.

Government of India has taken various initiatives in the field of AI. Some of the notable Innovative Initiatives are - Implementation of Artificial Intelligence Task Force, Establishment of NITI Aayog's National Strategy for Artificial Intelligence under \#AIFORALL, setting up of four Committees for AI under Ministry of Electronics and Information technology(MEITY) [4]. Some of State Governments of India have also taken enormous initiatives, such as establishment of Centre of Excellence for Data Science and Artificial Intelligence (CoE-DS\&AI) by Karnataka, Safe and Ethical Artificial Intelligence Policy 2020 and Face Recognition Attendance System by Tamil Nadu, AIPowered System for monitoring driving behaviour by West Bengal, AI System to fight agricultural risks by Maharashtra etc [5,6]. As with any other technology, AI brings with it a span of opportunities and challenges. Artificial Intelligence is not a One-Technology mechanism. But instead it is collection of constructs like Machine Learning, Natural Language Processing, Rule Based Expert Systems, Physical Robots and Robotic Process Automation. In this paper, we describe the potential that AI offers by elaborating on the various AI constructs which automate to ease aspects of health care and help to break the barriers to overcome the pandemic in the near future [7].

\section{Types of AI Constructs that support healthcare}

\subsection{Machine Learning}

Machine learning is a technique helps the machine to learn with algorithm to analyze the data based on the representation given by the end user. The action takes place in the machine learning approach based on the mathematical models and data set used in that. The approach which give more accuracy as the human wish is considered as the efficient approach. Some approaches will take more time to give an output but most of the end user need the accurate result with irrespective of time especially in medical fields.

\subsection{Medical imaging of Infected patient}

Machine Learning Algorithms were used in radiological imaging for detecting the disease symptoms. A new automatic model to detect COVID-19 symptoms made a deep impact to predict Covid patients using a chest X-ray image.

\subsection{Intelligent E-Platform to fight Covid}

Many Intelligent E-Platforms were implemented in India to detect and prevent the spread of the disease. For example, Dozee, a device created by Turtle Shell Technologies, India which produced this device and make it accessible by common people to monitor their health status in a continuous manner. If the person going to hospital they have to undergone many lab test for diagnosing the diseases which reduced by this simple device. We can place these device under their mattresses and monitor the individual health condition based on their heart rates, stress, oxygen and others. It provide an analysis report based 
on the received metrics to the smart phone which was linked with the devices and help to early detection of diseases before it reaches the severity. In COVID early detection of symptoms helps to achieve the highest rate of curable from the diseases. The decision making is the pure machine learning approach which makes this device far better than other monitoring device. One other important product helpful in this pandemic situation is the Milagrow Seagull. It is the robot used for vacuum cleaning process which includes anti-bacterial and anti-viral properties in their vacuum machine which reduce the spread of disease or hospitals where the patients are admitted. The cleaning process include the wet cleaning process but the machine itself make them dry in few minutes. This device shows the display in real time the progress of cleaning process.

In the pandemic period everyone want their belongings to be very clean and make themselves to live in a protective manner. CoronaOven is the device developed by Bengaluru based organization to clean the objects or equipment's which ever you bring with yourself or bought from outside using the ultra violet rays. The UV rays having the wavelength of $253.7 \mathrm{~nm}$ which is not harmful for the human and it available in different size which is easily available for any kind of individual house or organization. Apps like COVID locator, Medical Teleconsultation app, Call DOC, Well being volunteer, Test Yourself and Cobot-19 app are some of the notable Applications used Nationwide to fight the pandemic. Aarogya Setu is the smartphone app especially for tracking the spread of covid around India [8]. This was launched by the Government of India which available in eleven Indian languages to make this app usable by all peoples of India. The app provides the detail of affected patients around you in the specific area which you mentioned is helpful for the people to avoid from moving between the locations. It also provides the details of active cases and helps for the data analyst to predict the spread of disease which support the administrators take precautions to reduce the spread.

Staqu is the another AI based application developed by a start-up from Gurgaon has responded the people queries regarding the COVID. This start-up owned a video-based tracking setup to identify the spread of COVID and provide the contagion through the application which helps the needy peoples through remote monitoring and provide the solution for their current situation regarding their health condition. They provide an alert to the users for their daily hygiene activities to keep them safe against COVID. Another organization in India designed a remotely operable ventilator system which is inexpensive. The Big Bang Boom is the company which produced that and deliver around the primary health centers where there is no specific conditions not available to maintain the oxygen cylinder ventilator systems. This product really helpful for many remote locations in India where the frequent transport facilities was not feasible. The Defence Institute of Advanced Technology (DIAT) in Pune, Maharashtra has developed an AI based COVID-19 detection tool(Press Trust of India 2020g). The tool uses the chest X-rays of patients to identify COVID-19 infection. It will be particularly helpful for radiologists and also in telemedicine.

An IIT-Roorkee Professor has also developed similar software which can detect COVID-19 and measure its severity using X-ray scan of the suspected patient(Press Trust of India 2020e). An AI based voice tool has also been developed and designed by a professor and her students in Mumbai(Press Trust of India 2020d). This tool is able to detect COVID-19 through voice-based diagnosis using a smartphone app. The tool will detect COVID-19 based on the fact that the voice of COVID-19 patient is different from the healthy person as COVID-19 severely compromises the lungs and airways. Artificial 
Intelligence is able to detect these differences which a normal ear cannot. When a person speaks to the microphone on the app, this voice tool breaks down the voice in multiple parameters like frequency, noise distortion etc. The values of these parameters are then compared to normal person's values which help to detect COVID-19. This tool is being pilot tested by University of Tor Vergata in Rome and has yielded 98\% accurate results. The Norway India Partnership Initiative (NIPI) in collaboration with the Wadhwani Institute of Artificial Intelligence has developed an artificial intelligence (AI)-powered tool which enables identification of COVID-19 through cough sound analysis [6]. The tool will detect COVID-19 based on the fact that the cough of a COVID-19 patient is different from other coughs. Based on the reference coughing pattern, this AI powered tool will be able to detect COVID-19 as soon as the person coughs in front of the machine.

\subsection{Natural Language Processing}

With the rapid increase in the use of the Internet, sentiment analysis has become one of the most popular fields of Natural Language Processing (NLP). Using sentiment analysis, the implied emotion in the text can be mined effectively for different occasions. People are using social media to receive and communicate different types of information on a massive scale during COVID-19 outburst $[9,10]$. Mining such content to evaluate people's sentiments can play a critical role in making decisions to keep the situation under control. The sentiment analysis of tweets posted by Indian citizens has been performed using NLP and machine learning classifiers. From April 5, 2020 to April 17, 2020, a total of 12,741 tweets having the keywords "Indialockdown" were extracted. Data have been extracted from Twitter using Tweepy API, annotated using TextBlob and VADER lexicons, and preprocessed using the Natural Language Tool kit provided by the Python. Eight different classifiers have been used to classify the data. The experiment achieved the highest accuracy of $84.4 \%$ with LinearSVC classifier and unigrams.

\subsection{Expert Systems}

Artificial Intelligence-based Expert System models have emerged as one of the powerful weapons to fight against COVID-19 in India. Decision makers have used Computer Simulators to understand how COVID-19 situation will evolve over time. Many scientists and companies from India has contributed with very useful Innovations during this pandemic. Some noted among them are

- TCS(Tata Consultancy Services), has developed Machine-Learning Expert Models to predict the severity of the disease and identify at-risk populations across the country(KPMG 2020; Ghosh, Ghosh, and Chakraborty 2020; Shinjini 2020). For instance, TCS(Tata Consultancy Services) in collaboration with Pune-based Prayas Health Group has developed 'Digital twin' which is a Virtual Computerized Based Model to forecast the spread of COVID-19 in Urban districts in India.

- MyGov, world's largest citizen engagement platform launched by the Government of India, partnered with Amplify.ai, a company which deals in conversational AI technology with the aim to make people aware of COVID-19 and provide them real-time updates(Das 2020). The virtual assistant or chatbot also allows citizens to ask relevant questions and clear their doubts related to COVID-19. 
- The Indian Council of Medical Research (ICMR) in collaboration with the tech giant IBM has implemented the Watson Assistant, an AI-based query answering system, on its portal. The Watson Assistant responds to the queries on COVID-19 raised by front-line workers and also general public. The Watson Assistant works $24 * 7$ and responds both in English and Hindi(The Economic Times 2020) [11].

\subsection{Physical Robots and Robotic Process Automation}

AI based Robotic Technologies has also put its maximum efforts to fight against the deadly pandamic in the recent times. India, known for its outstanding brains in the field of Technology has proved its mark with remarkable development in Robotic Process Automation to fight the Virus. Some of the Innovative Technologies developed were as follows:

- 'Milagrow iMap 9', a robot designed for floor disinfection purposes which can navigate and sanitize floors without any human involvement was launched by Milagrow HumanTech(Press Trust of India 2020f).

- Garuda Aerospace, a Chennai based start-up, has developed an automated disinfecting Unmanned Aerial Vehicle (UAV) called "Corona-Killer 100" (a disinfectant spraying drone) . Garuda Aerospace has deployed 300 "Corona-Killer 100" drones for disinfection purposes across 26 cities in India.

- AI based tools such as drones and mobile applications have also helped in enforcing quarantine and maintaining social distancing. Drones are being used to monitor the movement of COVID-19 suspects in quarantine centers. They are being leveraged for video surveillance and enforcing social distancing purposes. These are particularly helpful in the red alert and containment zones and in public places where people gather in large numbers like banks, ration shops etc.

- The Kerala Government has initiated the use of robots 'KARMI-Bot' and 'Nightingale19'(Bhatia 2020; Zachariah 2020). These robots serve food and medicines to the COVID-19 patients, collect trash used by the patients, enable video call between patients and doctors or relatives and perform disinfection of the isolation ward. Other states like Tamil Nadu and Jaipur have also explored the use of robots. From monitoring temperature and sanitizing spaces to enabling video conferencing with doctors, these robots by Indian startups are helping minimize human intervention and paving the way for the use of technology in our fight against COVID-19.

- The autonomous mobile robot Invento C-Astra has come to use in April 2020 for sanitizing the common areas like hospitals, living rooms and infected hallways. It avoid the human interception in moving into the infected areas which greatly helpful for the frontline peoples.

- Another robot-based monitoring device called Mitra scans the patients who enters the hospital regarding their body temperature and pressure with the system to enter their personal details. This avoid the doctors and others to make a direct contact without knowing that what they really need or by which they are affected.

- Invento Robotics has come up with another robot called RoboDoc which was used to reduce the risk of contracting with the virus. This is virtual reality-based software which takes the doctor virtually to the patient environment and they can avoid the unwanted physical movement for monitoring purpose. It also get the details of patients including their temperature, and their food intaking information. 


\section{Conclusion}

Artificial intelligence has put in a great impact in medical field specially during the pandemic in the present times. It is a reality which has instantly come true to fight the disaster that we should face and encourage its progress and appearance. In this paper, we discuss various AI techniques that help in speeding up researches and assisting in the current COVID-19 crisis. Also, various expert, innovative techniques in introduced in a diverse country like India were emphasized. The various Artificial Intelligence constructs has helped in creating appropriate treatment regimens, counteraction methodologies, and medication and immunization advancement. Likewise, the NLP paved is way in sentiment analysis thereby mining such content to evaluate people's sentiments that can play a vital role in making decisions to keep the situation under control . Computer-based Artificial Intelligence is not only useful in the treatment of COVID-19, but additionally, for their appropriate medical check-ups. Further work will along these times need to address their desires with the goal that the improvement of AI is to serve patients to overcome the disaster.

\section{References}

[1] Kolker E, Özdemir V, Kolker E. How healthcare can refocus on its super-customers (patients, $n=1$ ) and customers (doctors and nurses) by leveraging lessons from amazon, uber, and watson. Omics: a journal of integrative biology. 2016 Jun 1;20(6):329-33.

[2] Huang C, Wang Y, Li X, Ren L, Zhao J, Hu Y, Zhang L, Fan G, Xu J, Gu X, Cheng Z. Clinical features of patients infected with 2019 novel coronavirus in Wuhan, China. The lancet. 2020 Feb 15;395(10223):497-506.

[3] Xu X, Chen P, Wang J, Feng J, Zhou H, Li X, Zhong W, Hao P. Evolution of the novel coronavirus from the ongoing Wuhan outbreak and modeling of its spike protein for risk of human transmission. Science China Life Sciences. 2020 Mar;63(3):457-60.

[4] NITI A. Government of India. National Strategy for Artificial Intelligence AIFORALL [Internet]. 2020 [updated 2020 Apr 23; cited 2020 Aug 20]. Available from: "https://niti.gov.in/.

[5] Tamil Nadu e-Governance Agency (TNeGA). TNeGA Announces with Pride That the 'Face Recognition Based Attendance System' Has Arrived. We Have Been Running. [Internet]. 2020 [updated 2019 Sep 3; cited 2020 July 3]. Available from: https://tnega.tn.gov.in/latestnews/29. .

[6] Kumar R. Bihar Nod to NIPI to Develop AI-Powered Tool to Track Covid-19 Suspects. Bihar Nod to NIPI to Develop AI-Powered Tool to Track Covid-19 Suspects [Internet]. 2020 [updated 2020 Apr 17; cited 2020 Sep 20]. Available from: https://www.hindustantimes.com/patna/bihar-nod-to-nipi-todevelop-aipowered-tool-to-track-covid-19-suspects/story-YDXNp1x8jLpfOrKup4Uh6K.html.

[7] Kulkarni, Viraj. 2020. "How India Is Using Artificial Intelligence to Combat COVID-19" [Internet]. 2020 [cited 2020 Sep 5]. Available from: https://www.theweek.in/news/sci-tech/2020/07/31/how-indiais-using- artificialintelligence-to-combat-covid-19.html.

[8] National Informatics Centre, Ministry of Electronics \& Information Technology, Government of India. 2020. "Aarogya Setu Mobile App.” Aarogya Setu Mobile App [Internet]. 2020 [updated 2020 Jan 15; cited 2020 May 5]. https://www.mygov.in/aarogya-setu-app/.

[9] Murdoch TB, Detsky AS. The inevitable application of big data to health care. Jama. 2013 Apr 3;309(13):1351-2.

[10] Dilsizian SE, Siegel EL. Artificial intelligence in medicine and cardiac imaging: harnessing big data and advanced computing to provide personalized medical diagnosis and treatment. Current cardiology reports. 2014 Jan 1;16(1):441.

[11] The Economic Times. 2020. ICMR Deploys IBM Watson Assistant to Empower Frontline Testing Facilities [Internet]. 2020 [updated 2019 Oct 20; cited 2020 July 23]. Available from: https://cio.economictimes.indiatimes.com/news/next- gentechnologies/icmr-deploys-ibm-watsonassistant-to-empower-frontline-testing facilities/75532811. 